\title{
Strongly driven resonant Auger effect treated by an open-quantum-system approach
}

\author{
Nina Rohringer ${ }^{*}$ \\ Max Planck Institute for the Physics of Complex Systems, 01187 Dresden, Germany and \\ Center for Free-Electron Laser Science, Max Planck Advanced Study Group, 2607 Hamburg, Germany \\ Robin Santra ${ }^{\dagger}$ \\ Center for Free-Electron Laser Science, DESY, 22607 Hamburg, Germany and \\ Department of Physics, University of Hamburg, 20355 Hamburg, Germany \\ (Received 22 May 2012; revised manuscript received 23 August 2012; published 31 October 2012)
}

\begin{abstract}
We present theoretical studies of a two-step resonant Auger process at high x-ray intensity. Tuning a short x-ray pulse to the initially closed resonant channel of the $1 s-2 p$ transition in singly ionized neon, the initially neutral neon target is valence ionized. Subsequently, the strong resonant $x$-ray field transfers an inner-shell electron to the created outer valence vacancy, thereby creating a core-excited state. The strong resonant coupling, giving rise to Rabi oscillations involving a core transition, results in a modification of the resonant Auger-electron spectral line profile. If the valence photoelectron remains unobserved, the system of the residual ion undergoing the resonant Auger decay can be treated by an open quantum system approach. The resonant Auger-electron spectral line shape is shown to be determined by an analog of the reduced density matrix that depends on two time arguments. The equations of motion of this reduced density matrix are derived and numerical results are presented, in support of the recent experimental verification [E. Kanter et al., Phys. Rev. Lett. 107, 233001 (2011)] of this nonlinear $\mathrm{x}$-ray optical effect.
\end{abstract}

DOI: 10.1103/PhysRevA.86.043434

PACS number(s): $32.80 . \mathrm{Hd}, 32.80 . \mathrm{Fb}, 32.70 . J z, 32.80 . \mathrm{Aa}$

\section{INTRODUCTION}

The recent start up of x-ray free electron laser (XFEL) sources [1-3] opened the opportunity to study fundamental interactions of femtosecond, high-intensity radiation with atoms in the x-ray regime [4-7]. A prominent example of nonlinear interaction is the strong, resonant coupling of a two-level system, inducing a coherent, oscillatory population transfer (Rabi oscillations) between the two energy levels. In the high-intensity $\mathrm{x}$-ray regime, the excitation of an inner-shell electron to a valence vacancy creates a core-excited state of short lifetime. In addition to stimulated emission, the core hole can undergo an Auger decay, resulting in damped Rabi oscillations. (Spontaneous emission is not important in the problem considered here.) The spectral line shape of the resonant Auger electron is modified in the strong-field limit [8-11], i.e., when the Rabi oscillation period is comparable to the Auger lifetime of the core hole. Another effect resulting in a change of the spectral line shape in the regular Auger process is the Autler-Townes Stark splitting of the final state, induced by strong resonant excitation of the final state by an x-ray field [12]. Similar effects due to strong resonant coupling are known in the optical regime, such as the modification of the photoelectron spectrum due to a strongly driven autoionizing resonance [13] or resonance fluorescence [14]. In a recent experiment, the broadening of the resonant Auger spectral line profile was demonstrated by resonant coupling of an initially inaccessible resonance in neon [6]. Here, we present the underlying theoretical model in detail, which was the basis of the interpretation of the experimental findings of Ref. [6].

In the studied system, the incident XFEL pulse, assumed to be linearly polarized along the $z$ direction, is tuned to

\footnotetext{
*nina.rohringer@asg.mpg.de

†robin.santra@cfel.de
}

the $1 s-2 p$ resonance of $\mathrm{Ne}^{1+}$ at $849 \mathrm{eV}$ photon energy (transition between configuration states $|2 p\rangle:=1 s^{2} 2 s^{2} 2 p^{5}$ to $|1 s\rangle:=1 s^{1} 2 s^{2} 2 p^{6}$ ). The XFEL beam is focused into a target of neon atoms, initially in their neutral ground state $\left|\Phi_{0}\right\rangle$. Since the XFEL photon energy is below the $K$ edge $(\approx 870 \mathrm{eV})$ and the first core-excited state $(\approx 867 \mathrm{eV})$ of the neutral neon atom, the pulse can initially only ionize the $2 s$ and $2 p$ shells (see schematics presented in Fig. 1). Once a valence hole in the $2 p_{z}$ orbital is created, the XFEL then resonantly couples the $1 s-2 p$ transition in $\mathrm{Ne}^{1+}$, giving rise to the resonant Auger electrons originating from core-excited $\mathrm{Ne}^{1+}$, which are observed in the experiment. The process, hence, involves a double-continuum of electronic states. The continuum electrons, however, are separated in energy, with the first continuum corresponding to the valence photoelectrons and the second continuum corresponding to that of the Auger electrons. The Auger process considered here is a real nonlinear process, even in the absence of strong Rabi oscillations, since the core-excited state is prepared by interaction with two photons. If the valence photoelectron remains unobserved, the residual singly-charged ion, undergoing stimulated absorption, emission, and Auger decay, can be treated as an open quantum system. The open system is described by a reduced ionic density matrix. Due to the loss of information by the unobserved valence photoelectron, this reduced density matrix generally does not describe a pure state. The loss of coherence is reflected in the spectral line shape of the observed Auger electron. We show that the Auger-electron line shape is governed by dynamic interference effects and is determined by an analog of the reduced density matrix (of the singly charged ion) that depends on two time arguments. The equations of motion of this object, describing temporal correlations of the ionic configuration and hence containing memory effects, will be derived. We present numerical results of the temporal evolution of the reduced density matrix and the Auger-electron line profile. Different 
1.Valence ionization 2.Strong resonant coupling
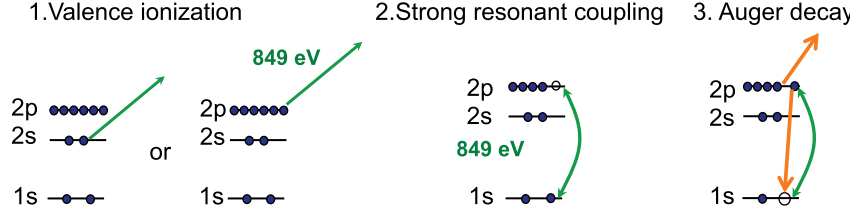

FIG. 1. (Color online) XFEL source is tuned to the $1 s-2 p$ resonance of singly ionized neon, at $849 \mathrm{eV}$. Initially, only valence shell ionization is possible, resulting in a hole either in the $2 s$ or $2 p$ shell. In case that a hole in the $2 p_{z}$ orbital is created, the XFEL then strongly couples the $1 s$ and $2 p$ shells, transferring population to the upper state. Once the core hole is created, Auger decay and stimulated emission compete.

assumptions for the driving $\mathrm{x}$-ray field will be compared, i.e., transform limited femtosecond pulses and pulses from a self-amplified spontaneous emission (SASE) XFEL source.

\section{THEORETICAL APPROACH}

In this section we introduce our method to calculate the resonant Auger-electron spectral line shape. We start out with the full quantum mechanical system and derive the expansion coefficients of the many-body wave function, expanded in configuration states of the atomic ground state, singly ionized neon plus continuum electron, and doubly charged neon plus electronic double-continuum. We then derive the expression for the Auger-electron line shape as a function of expansion coefficients of the states involving the double continuum. It turns out that the Auger spectrum can be calculated by an object, which is an analog of the reduced density matrix of the singly charged ion depending on two time arguments. Hence, for determining the Auger line shape, the system can be reduced to that of the singly charged ion. Subsequently, the equations of motion for this generalized reduced ionic density matrix are derived.

The Hamiltonian of our system is given by

$$
\hat{H}=\hat{H}_{0}+\hat{z} E(t),
$$

where $\hat{H}_{0}$ is the atomic part of the Hamiltonian in a mean-field approximation. The second term describes the interaction of the atom with the x-ray field in dipole approximation. The electric field of the $x$-ray radiation is written in terms of a slowly varying envelope $\varepsilon_{0}(t)$ and a phase $\phi(t)$ as

$$
E(t)=\frac{\varepsilon_{0}(t)}{2}\left[e^{i\left[\omega_{0} t-\phi(t)\right]}+e^{-i\left[\omega_{0} t-\phi(t)\right]}\right],
$$

where we assumed linear polarization of the field in $z$ direction. The carrier frequency $\omega_{0}=849 \mathrm{eV}$ is supposed to be tuned to the $1 s-2 p$ resonance of singly ionized neon.

\section{A. Expansion of the wave function}

In the second quantization, the ground state $\left|\Phi_{0}\right\rangle$ of a closed shell atom is then formally constructed by the action of singleparticle creation operators on the vacuum state by

$$
\left|\Phi_{0}\right\rangle=\prod_{i=1}^{N / 2} \hat{b}_{i \uparrow}^{\dagger} \hat{b}_{i \downarrow}^{\dagger}|0\rangle,
$$

where $\hat{b}_{i \uparrow}^{\dagger}\left(\hat{b}_{i \downarrow}^{\dagger}\right)$ are the fermionic creation operators, creating an electron in state $i=\left(n_{i}, l_{i}, m_{i}\right)$ with spin up (down). The active Hilbert space in our model is restricted to the ground-state configuration and a set of spin-singlet excited states of one- and two-particle excitations. The one-particle excited spin-singlet states are constructed by

$$
\left|\Phi_{i}^{a}\right\rangle:=\frac{1}{\sqrt{2}}\left(\hat{b}_{a \uparrow}^{\dagger} \hat{b}_{i \uparrow}+\hat{b}_{a \downarrow}^{\dagger} \hat{b}_{i \downarrow}\right)\left|\Phi_{0}\right\rangle .
$$

In the following $a, c$, and $d$ denote virtual orbitals, i.e., orbitals which are not occupied in the ground-state configuration, whereas $i, j$, and $k$ denote initially occupied orbitals. Doubly excited states are constructed along this line, by operation of another symmetric pair of creation and annihilation operators. The time-dependent atomic wave function is approximated by a superposition of the $\left|\Phi_{0}\right\rangle$, singly excited states $\left|\Phi_{i}^{a}\right\rangle$, and doubly excited states $\left|\Phi_{j k}^{c d}\right\rangle$ :

$$
\begin{aligned}
|\Psi, t\rangle= & \alpha_{0}(t) e^{-i E_{0} t}\left|\Phi_{0}\right\rangle+\sum_{i, a} \alpha_{i}^{a}(t) e^{-i E_{i}^{a} t}\left|\Phi_{i}^{a}\right\rangle \\
& +\sum_{j, k, c, d} \alpha_{j k}^{c d}(t) e^{-i E_{j k}^{c d} t}\left|\Phi_{j k}^{c d}\right\rangle .
\end{aligned}
$$

In the interaction picture the wave function $|\Psi, t\rangle_{I}:=$ $e^{i H_{0} t}|\Psi, t\rangle$ is then given by

$$
|\Psi, t\rangle_{I}=\alpha_{0}(t)\left|\Phi_{0}\right\rangle+\sum_{i, a} \alpha_{i}^{a}(t)\left|\Phi_{i}^{a}\right\rangle+\sum_{j, k, c, d} \alpha_{j k}^{c d}(t)\left|\Phi_{j k}^{c d}\right\rangle .
$$

The Schrödinger equation in the interaction picture then determines the time evolution of the expansion coefficients. Neglecting field induced transitions between virtual orbitals one gets

$$
\begin{gathered}
i \dot{\alpha}_{0}=\sum_{i, a} \alpha_{i}^{a} e^{i\left(\epsilon_{i}-\epsilon_{a}\right) t} E(t) \sqrt{2} z_{i, a}, \\
i \dot{\alpha}_{i}^{a}=\alpha_{0}(t) e^{i\left(\epsilon_{a}-\epsilon_{i}\right) t} E(t) \sqrt{2} z_{a, i} \\
-\sum_{j} e^{i\left(\epsilon_{j}-\epsilon_{i}\right) t} \alpha_{j}^{a} E(t) z_{j, i}-i \frac{\Gamma_{i}}{2} \alpha_{i}^{a}, \\
i \dot{\alpha}_{j k}^{c d}=\sum_{a} \alpha_{1 s}^{a} e^{i\left(E_{j k}^{c d}-E_{1 s}^{a}\right) t}\left|\Phi_{j k}^{c d}\right| \hat{V}_{C}\left|\Phi_{1 s}^{a}\right\rangle .
\end{gathered}
$$

where $z_{i, a}$ is the dipole matrix element of the single-particle transition between orbitals $i$ and $a$, and $\epsilon_{i}$ denotes the one-particle orbital energy. To account for the decay of the inner-shell hole through the Auger process, we introduced a phenomenological decay term with decay width $\Gamma_{i}$, where $\Gamma_{1 s}=0.27 \mathrm{eV}$ [15] denotes the decay width of the $1 s$ hole in neon; all other $\Gamma_{i}=0$. This effective decay term can be derived by the Wigner-Weisskopf method [16]. In Eq. (9) we assumed that the double continuum states $\left|\Phi_{j k}^{c d}\right\rangle$ are populated only by Auger decay, mediated by the Coulomb interaction $\hat{V}_{C}$, i.e., we neglected the occupation of that channel by direct double ionization of the valence shell.

\section{B. Calculation of resonant Auger-electron spectral line shape}

The Auger-electron spectral line shape is determined by the asymptotic occupations of the doubly valence-excited 
states $\alpha_{j k}^{c d}(t)$, which evolve according to Eq. (9). We assume that the photoelectrons are energetically distinguishable from the Auger electrons. The index $d$ in Eq. (9) refers to the Auger electron, whereas $a$ and $c$ refer to the photoelectrons. We disregard any interaction of the photoelectron with the Auger electron; hence $c=a$ in Eq. (9). This is justified, since the photoelectron, resulting from valence ionization, has a high energy and a coupling (postcollision interaction) of the photoelectron and Auger electron can be neglected. In the following we only consider the strongest resonant Auger decay channel $\left({ }^{1} D\right)$, involving both participating electrons from the $2 p$ shell. Coupling of the different decay channels by the field is neglected. With these assumptions we arrive at

$$
i \dot{\alpha}_{j k}^{a d}=e^{i\left(E_{j k}^{a d}-E_{1 s}^{a}\right) t} \sqrt{\frac{\Gamma_{j k}}{2 \pi}} \alpha_{1 s}^{a}=e^{i\left(\epsilon_{d}-\epsilon_{j}-\epsilon_{k}+\epsilon_{1 s}\right) t} \sqrt{\frac{\Gamma_{j k}}{2 \pi}} \alpha_{1 s}^{a},
$$

where we introduced the partial Auger width $\Gamma_{j k}=$ $2 \pi\left|\left\langle\Phi_{j k}^{a d}\left|\hat{V}_{C}\right| \Phi_{1 s}^{a}\right\rangle\right|^{2}$, and the Auger channel is specified by the quantum numbers $j, k$. The index $d$ refers to the energynormalized continuum wave function and satisfies energy conservation in the Auger decay, i.e., the Auger electron carries the energy shift between the $1 s$-hole state and the final dicationic state $j, k$. Strictly, the energy of the state $d$ in Eq. (9) is not constant, but we neglect the dependence of the matrix element $\left\langle\Phi_{j k}^{c d}\left|\hat{V}_{C}\right| \Phi_{1 s}^{a}\right\rangle$ on the state $d$. These two assumptions then allow us to introduce the decay width $\Gamma_{j k}$ of the core-excited state. The total decay width of the $1 s$-hole state is given by $\Gamma_{1 s}=\sum_{j, k} \Gamma_{j, k}$. The Auger-electron line shape is then determined by the asymptotic limit of the expansion coefficients, which after formal integration of the differential equations results in

$$
\begin{aligned}
P_{j, k}(\epsilon)= & \sum_{a}\left|\alpha_{j k}^{a d}(t \rightarrow \infty)\right|^{2} \\
= & \frac{\Gamma_{j k}}{2 \pi} \int_{-\infty}^{\infty} d t \int_{-\infty}^{\infty} d t^{\prime} e^{i\left(\epsilon-\epsilon_{j}-\epsilon_{k}+\epsilon_{1 s}\right)\left(t-t^{\prime}\right)} \\
& \times \sum_{a} \alpha_{1 s}^{a}(t) \alpha_{1 s}^{a *}\left(t^{\prime}\right) .
\end{aligned}
$$

The partial Auger width $\Gamma_{j k}$ enters only as a normalization factor in Eq. (11). The quantity

$$
\rho_{1 s, 1 s}\left(t, t^{\prime}\right):=\sum_{a} \alpha_{1 s}^{a}(t) \alpha_{1 s}^{a *}\left(t^{\prime}\right)
$$

appearing in Eq. (11) can be interpreted as an analog of the reduced density matrix of the subsystem of the singly charged ion and depends on two time arguments. Equation (11) highlights the fact that the Auger-electron spectral line shape can be determined by eliminating both continua related to the photoelectron and the Auger electron, at the expense of introducing an object related to the reduced ionic density matrix which treats memory effects and depends on two time arguments.

We want to stress that there are several strategies to calculate the resonant Auger-electron line shape. One approach would be to deduce the wave-function expansion coefficients of the closed system by solving Eqs. (7) through (9). Then one would calculate the photo and Auger-electron coincidence spectrum, from which the Auger-electron spectrum can be determined. This traditional strategy was, for example, applied in Ref. [17]. Another possibility is to solve for the expansion coefficients determined by Eqs. (7) and (8). Subsequently, one calculates the Auger-electron spectrum, given by Eq. (11), an expression in which the Auger-electron continuum has been already eliminated. This approach involves the explicit treatment of the photoelectron continuum. Alternatively, one can treat the system as an open quantum system of the residual ion, eliminating both the photo and the Auger-electron continuum. We decided to adopt this approach. Equation (11) shows that the Auger-electron line shape can be determined by eliminating the unobserved continuum associated with the photoelectron, at the cost that the time evolution of the two-time analog of the reduced density matrix, defined by Eq. (12), has to be determined. Note that the usual reduced density matrix

$$
\rho_{1 s, 1 s}(t):=\sum_{a} \alpha_{1 s}^{a}(t) \alpha_{1 s}^{a *}(t)
$$

does not carry sufficient information of the ionic subsystem, in order to determine the Auger line shape. The object $\rho_{1 s, 1 s}\left(t, t^{\prime}\right)$ can be interpreted in terms of a second-order correlation function (or the expectation value of the propagator in the ionic subsystem), related to the probability that the ionic subsystem has a hole in the $1 \mathrm{~s}$ shell at time $t^{\prime}$ under the condition that it had a hole in the $1 s$ shell at time $t$. More than that, the object $\rho_{1 s, 1 s}\left(t, t^{\prime}\right)$ is complex valued [unlike $\rho_{1 s, 1 s}(t)$ ] and hence carries a phase. The Auger-electron line shape is therefore determined by a dynamic interference process. In other words, whenever the neon ion is in the core-excited state, it can decay by an Auger process. The Auger-electron spectrum is given by a coherent sum of spectra emitted at times for which the ionic system is inverted. We opt for the open quantum system approach, since it highlights the analogy to the resonantly driven two-level system. In the following, we derive the equations of motion for the generalized reduced density matrix, which determines the Auger-spectral line profile.

\section{Equations of motion}

In principle, the Auger line shape Eq. (11) can be determined by solving the equations of motion for the expansion coefficients Eqs. (7) and (8) by treating the photoelectron continuum explicitly. We opt for an alternative description and treat the problem by an open quantum system approach. As shown in the previous section, the residual ion undergoing Auger decay can be characterized by a two-time analog of the reduced density matrix defined in Eq. (12). This highlights the parallels with optically driven few level systems treated by the usual density-matrix approach. The problem can hence be reduced to that of an open quantum system, solely determined by the evolution of the reduced ionic two-time density matrix

$$
\rho_{i, j}\left(t, t^{\prime}\right)=\sum_{a} \alpha_{i}^{a}(t) \alpha_{j}^{a *}\left(t^{\prime}\right), \quad i, j=1 s, 2 p_{z} .
$$

Making use of the expression in Eq. (14) and the time evolution of the coefficients Eqs. (7) and (8) we can derive the equations determining the temporal evolution of the reduced ionic density matrix. Applying the rotating wave approximation, 
after some lengthy but straightforward calculations sketched in the Appendix one arrives at

$$
\begin{aligned}
\frac{\partial}{\partial t} \rho_{2 p_{z}, 2 p_{z}} & =\frac{\sigma_{2 p_{z}}}{2} J(t) p_{0}(t)+i e^{-i \delta t} \frac{R^{*}(t)}{2} \rho_{1 s, 2 p_{z}}, \\
\frac{\partial}{\partial t^{\prime}} \rho_{2 p_{z}, 2 p_{z}} & =\frac{\sigma_{2 p_{z}}}{2} J\left(t^{\prime}\right) p_{0}\left(t^{\prime}\right)-i e^{i \delta t^{\prime}} \frac{R\left(t^{\prime}\right)}{2} \rho_{2 p_{z}, 1 s}, \\
\frac{\partial}{\partial t} \rho_{1 s, 1 s} & =-\frac{\Gamma_{1 s}}{2} \rho_{1 s, 1 s}+i e^{i \delta t} \frac{R(t)}{2} \rho_{2 p_{z}, 1 s}, \\
\frac{\partial}{\partial t^{\prime}} \rho_{1 s, 1 s} & =-\frac{\Gamma_{1 s}}{2} \rho_{1 s, 1 s}-i e^{-i \delta t^{\prime}} \frac{R^{*}\left(t^{\prime}\right)}{2} \rho_{1 s, 2 p_{z}}, \\
\frac{\partial}{\partial t} \rho_{2 p_{z}, 1 s} & =i e^{-i \delta t} \frac{R^{*}(t)}{2} \rho_{1 s, 1 s}, \\
\frac{\partial}{\partial t^{\prime}} \rho_{2 p_{z}, 1 s} & =-\frac{\Gamma_{1 s}}{2} \rho_{2 p_{z}, 1 s}-i e^{-i \delta t^{\prime}} \frac{R^{*}\left(t^{\prime}\right)}{2} \rho_{2 p_{z}, 2 p_{z}}, \\
\frac{\partial}{\partial t} \rho_{1 s, 2 p_{z}} & =-\frac{\Gamma_{1 s}}{2} \rho_{1 s, 2 p_{z}}+i e^{i \delta t} \frac{R(t)}{2} \rho_{2 p_{z}, 2 p_{z}}, \\
\frac{\partial}{\partial t^{\prime}} \rho_{1 s, 2 p_{z}} & =-i e^{i \delta t^{\prime}} \frac{R\left(t^{\prime}\right)}{2} \rho_{1 s, 1 s},
\end{aligned}
$$

where we omitted the time dependence of the density matrix elements. We introduced the detuning $\delta=\epsilon_{2 p_{z}}-\epsilon_{1 s}-\omega_{0}$ and the photoionization cross section $\sigma_{2 p_{z}}=4.2 \times 10^{-21} \mathrm{~cm}^{2}$ $[18,19]$ for ionization of the $2 p_{z}$ orbital and the complex Rabi frequency

$$
R(t)=z_{1 s, 2 p_{z}} \varepsilon_{0}(t) e^{i \phi(t)},
$$

with $z_{1 s, 2 p_{z}}=0.0554$ a.u. $[18,19]$. The cycle averaged flux is given by

$$
J(t)=\frac{c}{4 \pi \omega_{0}}\left\langle E^{2}(t)\right\rangle=\frac{c}{8 \pi \omega_{0}} \epsilon_{0}^{2}(t) .
$$

The ground-state occupation $p_{0}(t)$ is determined by

$$
\dot{p}_{0}(t)=-\sigma_{\text {tot }} J(t) p_{0}(t),
$$

where $\sigma_{\text {tot }}=2.5 \times 10^{-20} \mathrm{~cm}^{2}[18,19]$ denotes the total valence photoionization cross section of neon at frequency $\omega_{0}$. The occupation probabilities of the holes $1 s$ and $2 p_{z}$ are given by the diagonal density matrix elements of the reduced density matrix at equal time arguments

$$
\rho_{i, j}(t):=\rho_{i, j}(t, t), \quad i, j=1 s, 2 p_{z} .
$$

Describing an open quantum system, the norm $N(t)=$ $\sum_{i} \rho_{i, i}(t, t)$ of the reduced density matrix is not conserved. Initially, the population sits in the ground state $p_{0}(0)=1$ and $N(0)=0$. As ionization of the $2 p_{z}$ orbital proceeds, the ionic density matrix gets populated. Resonant coupling of the $1 s$ and $2 p_{z}$ orbitals by the electric field then results in occupation of the $1 s$-hole states and the buildup of the coherence terms $\rho_{1 s, 2 p_{z}}$. Part of the ionization of the ground state results from the $2 s, 2 p_{x}$, and $2 p_{y}$ channels, which are not of interest to the considered processes and are loss terms. Equations (15) and (18) are solved numerically. Due to the symmetry relation $\rho_{i, j}\left(t, t^{\prime}\right)=\rho_{j, i}^{*}\left(t^{\prime}, t\right)$, the two-dimensional time integration of Eqs. (15) and (18) can be restricted to the symplex $t \in\left[0, t_{\max }\right], t^{\prime} \in\left[t, t_{\max }\right]$.

\section{NUMERICAL RESULTS AND DISCUSSION}

\section{A. Gaussian temporal profile}

As a first example, we study the effect of a coherent, transform limited pulse on the resonant Auger line shape. The intensity envelope is supposed to be Gaussian, with a pulse duration of $3 \mathrm{fs}$ full width at half maximum (FHWM). Figure 2 shows the evolution of the density matrix elements and the ground-state occupation as a function of time for a fluence of 350 a.u., corresponding to $1.25 \times 10^{11}$ photons per $\mu \mathrm{m}^{2}$. At this intensity, depletion of the ground state is not saturated; roughly $30 \%$ of the ground state get depopulated. The occupation probabilities of the $2 p_{z}$ - and $1 s$-hole states are plotted in Fig. 2(b). The resonant coupling of $1 s$ to $2 p$ is strong and the occupation probabilities of the two hole states follow each other closely. The Auger decay and the resonant coupling happen fast compared to the valence ionization time, so that oscillations (Rabi oscillations) in the occupation probabilities are not visible on the scale of the occupations. Plotting the population inversion $\rho_{1 s, 1 s}(t)-\rho_{2 p_{z}, 2 p_{z}}(t)$ or the coherence $\rho_{1 s, 2 p_{z}}(t)$, the damped Rabi oscillations are more visible
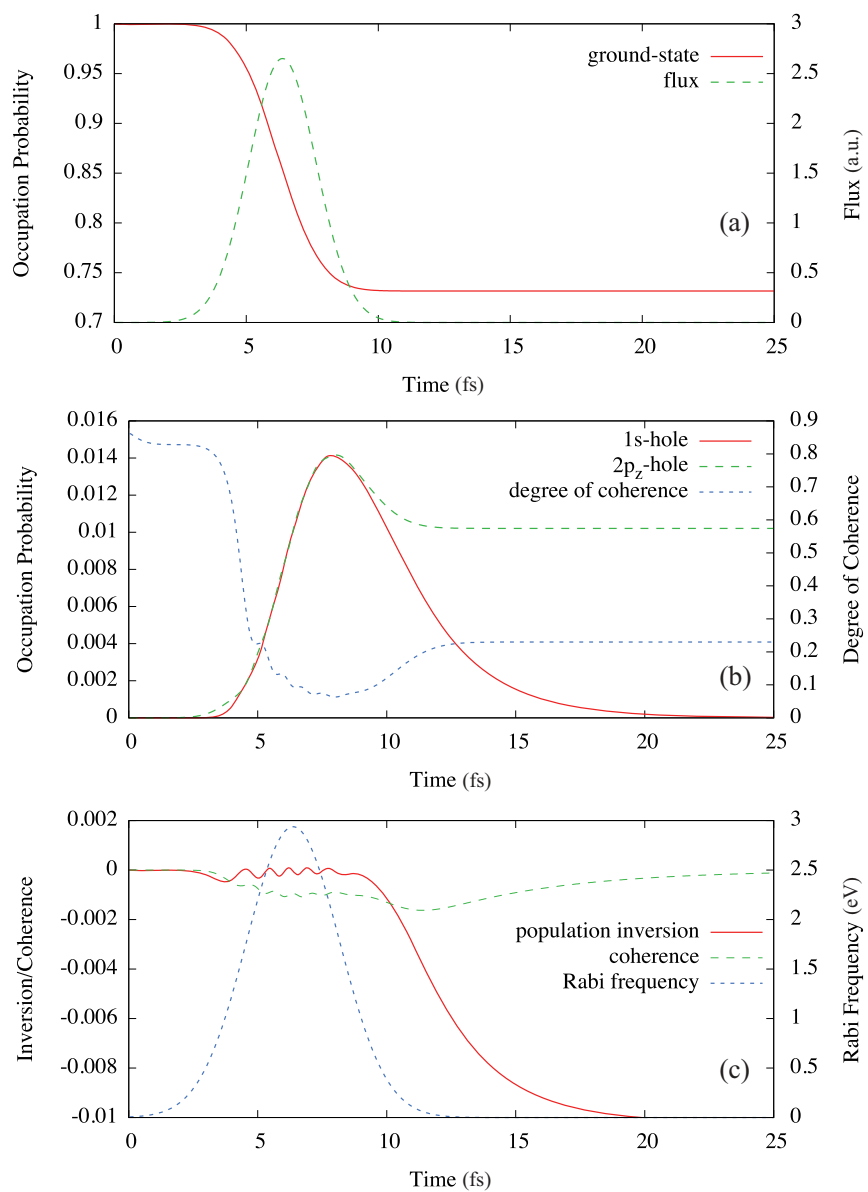

FIG. 2. (Color online) (a) Ground-state occupation and flux as a function of time. (b) Time evolution of the occupation probability of the $1 s$ and $2 p_{z}$ holes $\left[\rho_{1 s, 1 s}(t)\right.$ and $\rho_{2 p_{z}, 2 p_{z}}(t)$, respectively] and the degree of coherence. (c) Temporal evolution of the occupation inversion $\rho_{1 s, 1 s}(t)-\rho_{2 p_{z}, 2 p_{z}}(t)$, the imaginary part of the coherence $\rho_{1 s, 2 p_{z}}(t)$, and the Rabi frequency. The input pulse is a Gaussian pulse of $3 \mathrm{fs}$ FHWM, assuming a fluence of 350 a.u. 


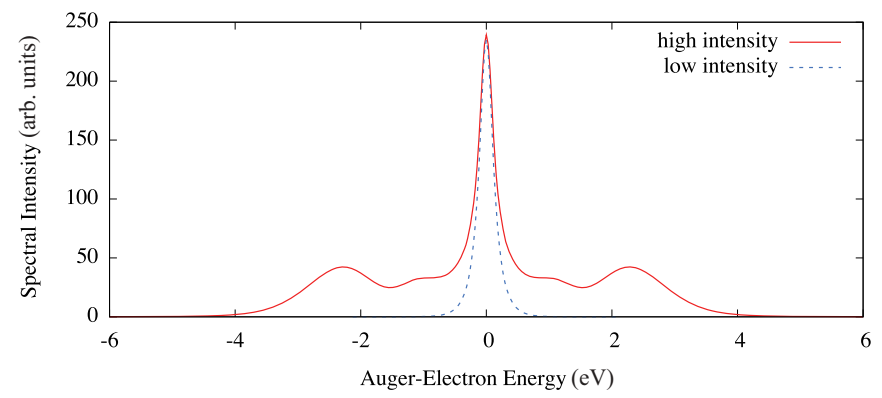

FIG. 3. (Color online) Auger-electron spectral line shape for a Gaussian pulse of $3 \mathrm{fs}$ FWHM and a fluence of 350 a.u. (red solid curve) and 0.5 a.u. (blue dashed curve). The spectra were rescaled, so that the peak strengths are comparable.

[see Fig. 2(c)]. In the considered case, the system undergoes nearly five Rabi cycles. Hence Auger decay and stimulated emission are competing, which has an imprint on the Augerelectron line shape, shown in Fig. 3. The line profile develops side lobes and is considerably broader than the spectrum at low intensity, which has a FHWM of $0.27 \mathrm{eV}$, in accordance with the unaltered Auger width in the perturbative limit. The regular structure with side peaks can be interpreted in terms of dynamic interference. Due to the oscillatory dependence of the upper state occupation, Auger emission temporally peaks at the occupation maxima, corresponding to a temporal grating. Since the Rabi frequency changes as a function of time, due to the intensity variation of the pulse, the temporal maxima are not equidistant in time, giving rise to a complex interference pattern. For a flat-top pulse, the pattern results in a center symmetric triple-peak structure, with an energy spacing of the peaks given by the Rabi frequency defined by the constant field.

During the evolution, the ionic system maintains only a limited degree of coherence, defined by

$$
C(t):=\frac{\left|\rho_{1 s, 2 p_{z}}(t)\right|}{\sqrt{\rho_{1 s, 1 s}(t) \rho_{2 p_{z}, 2 p_{z}}(t)}} .
$$

The degree of coherence defined in Eq. (20), or degree of purity, measures the deviation of the two-level system from a pure state [20]. If the system is in a completely coherent superposition of the hole states $|1 s\rangle$ and $\left|2 p_{z}\right\rangle$, then one can use a Schrödinger ket $c_{1 s}(t)|1 s\rangle+c_{2 p_{z}}(t)\left|2 p_{z}\right\rangle$ to describe the state. The corresponding density matrix has offdiagonal elements $c_{1 s}(t) c_{2 p_{z}}^{*}(t)$ and $c_{2 p_{z}}(t) c_{1 s}^{*}(t)$. Therefore, the coherence as defined in Eq. (20) would be one. On the other hand, if the system is in a completely incoherent superposition of $|1 s\rangle$ and $\left|2 p_{z}\right\rangle$, a Schrödinger ket cannot be used at all to describe the state of the system, and the off-diagonal elements of the density matrix vanish. Hence the degree of coherence is zero.

The initial degree of coherence, as can be seen in Fig. 2(b), is close but not equal to one and evolves as a function of time. It is determined by the specific pulse shape of the system and is determined by a $0 / 0$ limit, since both the off-diagonal and diagonal matrix element of the density matrix evolve from zero. For a constant electric field envelope the initial value of coherence is $\frac{\sqrt{3}}{2}$, the value reached in our numerical calculation. In the considered case, the singly charged ion is produced by valence ionization of the neutral on a time scale that is slow compared to the Rabi cycling time of the system and the pulse duration (the valence photoionization cross section is small compared to the resonant inner-shell coupling strength). Once the $2 p_{z}$-hole states are created by photoionization, they undergo fast Rabi oscillations. Hole states created at later times are not necessarily in phase with the wave packet created at earlier times. This loss of coherence of the quantum state is characterized by the limited degree of coherence of the system and is also reflected in the line shape of the emitted Auger electron. Along similar lines, we recently studied the degree of coherence of a valence spin-orbit wave packet created during the course of strong-field ionization with an optical laser pulse [21]. The degree of coherence of the electronic wave packet was recently measured in an experiment on krypton ions created by a few-cycle pulse [20]. The two distinct time scales governing dephasing of the density matrix in that example were given by the spin-orbit period and the pulse duration of the optical laser field.

Figure 4(a) shows the total Auger-electron yield as a function of fluence, in comparison to the total photoelectron yield and the $2 p_{z}$ photoelectron yield. Since the production of an Auger electron requires two photons in the low fluence limit, where perturbation theory is applicable, the total Auger-electron yield shows a quadratic dependence on the
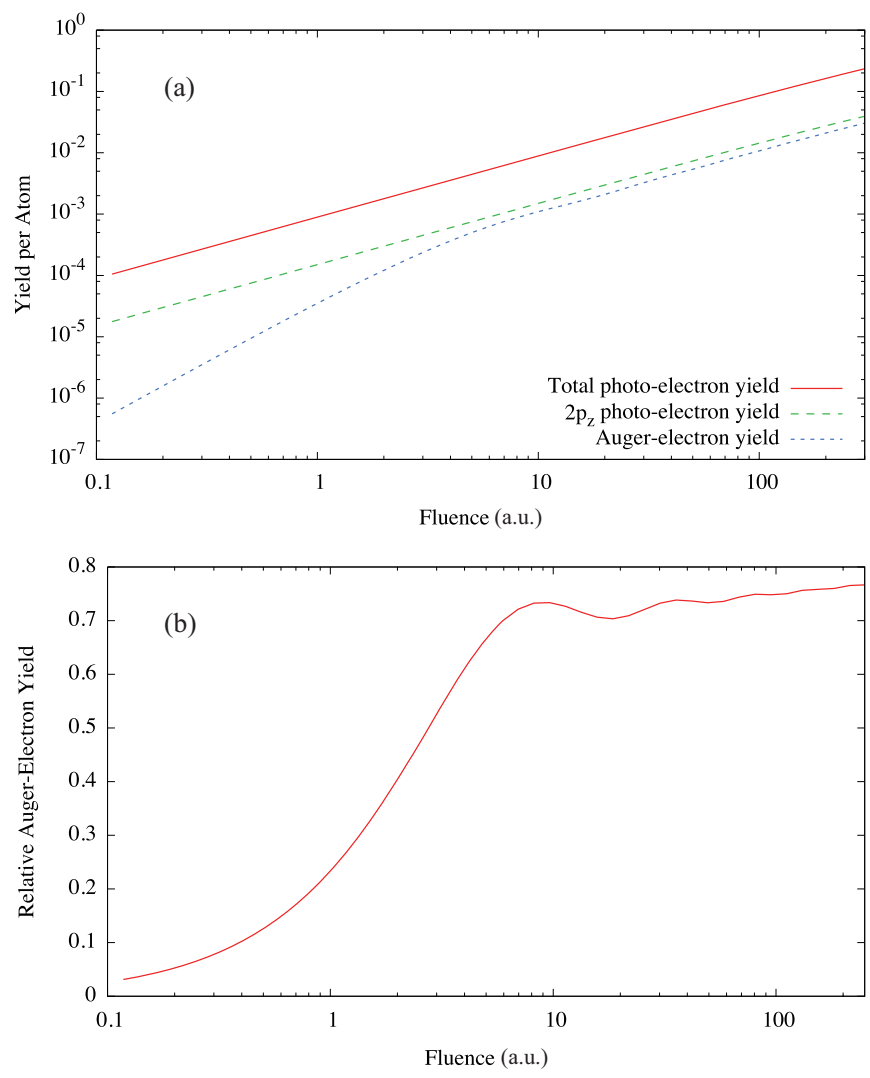

FIG. 4. (Color online) (a) Total photoelectron, $2 p$ photoelectron, and Auger-electron yield as a function of fluence for a Gaussian pulse of 3 fs duration. In the perturbative limit, i.e., when the resonant $1 s-2 p$ transition is not saturated, the Auger-electron yield shows a nearly quadratic dependence on the fluence. (b) Ratio of the Auger-electron yield to the total $2 p_{z}$ photoelectron yield as a function of fluence. 
fluence. In the region of strong resonant coupling, this is no longer the case and the yield grows nearly linearly with increasing fluence. The small deviations from the linear behavior are better visible in the relative Auger-electron yield. Figure 4(b) shows the ratio of the total Auger-electron yield and the total $2 p_{z}$ photoelectron yield as a function of fluence. At low fluence, the relative Auger-electron yield is small, since the production of the $1 s$ hole by resonant excitation into the $2 p$ shell is not saturated, reflecting the quadratic dependence of the Auger-electron yield. In analogy to direct resonant coupling of the $1 s-3 p$ resonance [8], the relative Auger-electron yield features oscillations at higher fluence. The minima of these oscillations lie at fluences corresponding to multiples of $2 \pi$ pulses, i.e., pulses which drive a given number of complete Rabi cycles (population transfer to the $1 s$ hole state and back to the $2 p_{z}$ hole state).

\section{B. SASE pulses of limited temporal coherence}

Presently, x-ray FELs are based on self-amplification of spontaneous emission (SASE) and their spectral and temporal intensity distribution is very noisy [22,23]. The pulses have finite temporal coherence. In the case of the LCLS, the temporal coherence was recently inferred from measurements in the hard x-ray regime [24] at around $8 \mathrm{keV}$ photon energy. Unfortunately, no measurements are available in the low x-ray energy regime around $850 \mathrm{eV}$. According to the experimentally measured photoelectron spectrum [6], we assume a bandwidth of $6 \mathrm{eV}$ at $850 \mathrm{eV}$, which would result in a coherence time of 0.2 fs, assuming FEL operation in the nonsaturated gain region [22,23]. To study the influence of these pulses of limited temporal coherence on the resonant Auger spectrum, we simulate a stochastic ensemble of SASE pulses assuming noise with a Gaussian spectral function with a width equal to the SASE gain bandwidth (for details, see $[8,25,26]$ ). We assume a Gaussian intensity envelope of 8.5 fs FWHM for the ensemble average of the SASE pulses. Figure 5 shows the temporal evolution of the occupancies of the relevant states for a typical representative of the SASE ensemble. Shown are results for a fluence of 350 a.u., corresponding to the conditions at the focus maximum of the experiment [6]. The ground-state occupation, shown in Fig. 5(a), follows the intensity spikes of the SASE pulse. Also the occupation probabilities of the $1 s$ and $2 p_{z}$ hole states and the degree of coherence, shown in Fig. 5(b), feature a noisy structure. The resulting Auger spectral line shape for a single representative of the SASE ensemble is shown in Fig. 6(a) for high and low fluence. The single-pulse profiles have several spikes and are substantially broadened at high fluence, i.e., where Rabi flopping is present. This becomes more apparent in the average over the SASE ensemble, shown in Fig. 6(b). At low intensity, the line shape is Lorentzian with the width given by the Auger width of $0.27 \mathrm{eV}$. The averaged line shape at high $\mathrm{x}$-ray intensity is considerably broader. There are no regular sidebands visible, in contrast to the case for a transform limited pulse. The spectral profile is characterized by broad tails, which also have been predicted for resonance fluorescence with stochastic light of Gaussian statistics [27]. To compare with experiments, integration over the spatial beam intensity profile is necessary, which typically flattens the broad tails of the high-intensity contributions to the
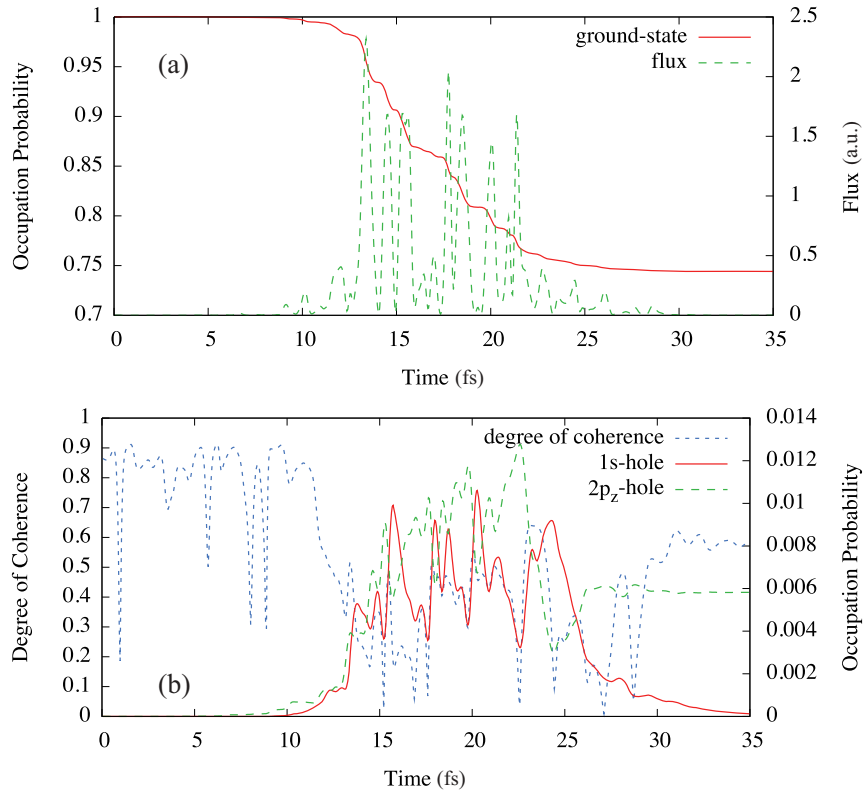

FIG. 5. (Color online) (a) Ground-state occupation and flux as a function of time. (b) Time evolution of the occupation probability of the $1 s$ and $2 p_{z}$ hole $\left[\rho_{1 s, 1 s}(t)\right.$ and $\rho_{2 p_{z}, 2 p_{z}}(t)$, respectively] and the degree of coherence.

spectrum. Nevertheless, a broadening of the Auger line was observed (see Ref. [6] for comparison to experimental data). Similar changes of emission spectra due to stochastic fields have been studied in resonance fluorescence with intensity and phase fluctuating optical laser pulses [28-32], or in the ac Stark effect in resonantly driven three-level systems [33]. A
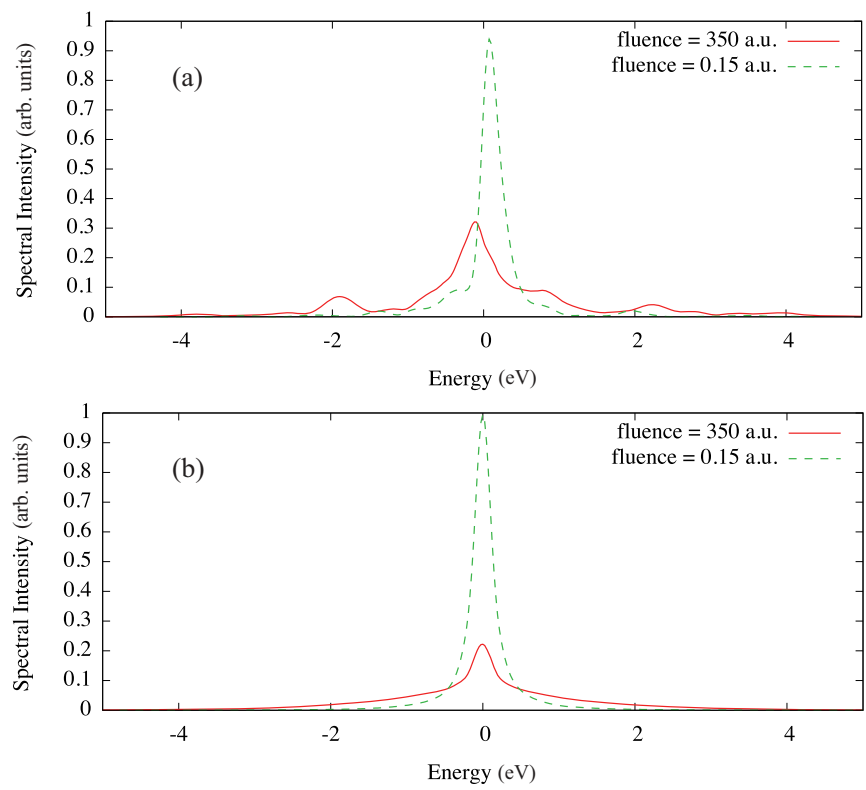

FIG. 6. (Color online) (a) Auger-electron spectral line profile for low (green dashed line) and high (red solid line) fluence for a single SASE pulse. (b) Spectra for an ensemble of 500 SASE pulses. The spectra are normalized to have the same integral. The SASE pulses are constructed in such a way that the ensemble averaged pulse is a Gaussian of pulse duration of $8.5 \mathrm{fs}$. 
good collection of references on systems resonantly driven by stochastic fields can be found in [34].

\section{CONCLUSIONS}

We discussed the resonant Auger effect of a strongly driven, open quantum system. Neon is subjected to a strong x-ray pulse, tuned on resonance of the $1 s-2 p$ transition of singly ionized neon. In a first step, the neon atom is valence-ionized by the $\mathrm{x}$-ray pulse. If the valence hole is created in the $2 p_{z}$ orbital, the $\mathrm{x}$-ray pulse subsequently drives a population transfer from the $1 s$ to the $2 p$ shell, which is followed by an Auger decay of the $1 s$ hole state. The photoelectron remains unobserved. Therefore, the system can be treated as an open quantum system, describing the residual ion undergoing stimulated absorption, emission, and Auger decay. We showed that the Auger-electron spectral line shape is determined by a two-time analog of the reduced ionic density matrix. Equations of motion for this reduced density matrix have been presented. The strong resonant coupling and the induced Rabi oscillations are manifested in a pronounced change of the Auger-electron line shape. For a transform-limited Gaussian-shaped temporal pulse, the Auger spectral line shape at high x-ray fluence develops sidebands, which can be interpreted in terms of dynamic interference. For a stochastic radiation field, which describes the case of an ensemble of SASE x-ray free-electron laser pulses, a considerable broadening of the Auger spectral line shape is predicted, which was observed in a recent experiment [6].

\section{ACKNOWLEDGMENTS}

We thank E. Kanter, S. Southworth, B. Krässig, and L. Young for discussions.

\section{APPENDIX: DERIVATION OF THE EQUATIONS OF MOTION}

This appendix gives details on how the equations of motion (15) and (18) for the reduced two-time density matrix and the ground-state occupation are derived. The evolution of the ground state occupation $p_{0}(t)=\left|\alpha_{0}(t)\right|^{2}$ in terms of the expansion coefficient $\alpha_{0}$ is determined by

$$
\dot{p}_{0}(t)=2 \operatorname{Re}\left[\dot{\alpha}_{0}(t) \alpha_{0}^{*}(t)\right]
$$

which, by inserting the evolution of the ground-state expansion coefficient of Eq. (7), gives

$\dot{p}_{0}(t)=2 \operatorname{Re}\left\{-i \alpha_{0}^{*}(t) \sum_{i, a} \alpha_{i}^{a}(t) e^{i\left(\epsilon_{i}-\epsilon_{a}\right) t} E(t) \sqrt{2} z_{i, a}\right\}$.

The next step is to insert the formal integration of Eq. (8) for $\alpha_{i}^{a}(t)$, which yields

$$
\begin{aligned}
\dot{p}_{0}(t)= & -2 \operatorname{Re}\left\{\alpha_{0}^{*}(t) \sum_{i, a} e^{i\left(\epsilon_{i}-\epsilon_{a}\right) t} E(t) \sqrt{2} z_{i, a} \int_{-\infty}^{t} d t^{\prime} e^{-\frac{\Gamma_{i}}{2}\left(t-t^{\prime}\right)}\right. \\
& \times E\left(t^{\prime}\right)\left[\alpha_{0}\left(t^{\prime}\right) e^{i\left(\epsilon_{a}-\epsilon_{i}\right) t^{\prime}} \sqrt{2} z_{a, i}\right. \\
& \left.\left.-\sum_{j} e^{i\left(\epsilon_{j}-\epsilon_{i}\right) t^{\prime}} \alpha_{j}^{a}\left(t^{\prime}\right) z_{j, i}\right]\right\} .
\end{aligned}
$$

In general, after integration over the continuum $\left(\sum_{a}\right)$ the second term of the integrand will give rise as a function of $t$ to a rapidly oscillating contribution to the photoabsorption rate. This second term will therefore be neglected in the following. We therefore get

$$
\begin{aligned}
\dot{p}_{0}(t)= & -4 \operatorname{Re}\left\{\alpha_{0}^{*}(t) E(t) \sum_{i, a}\left|z_{a, i}\right|^{2}\right. \\
& \left.\times \int_{-\infty}^{t} d t^{\prime} e^{i\left(\epsilon_{i}-\epsilon_{a}\right)\left(t-t^{\prime}\right)} e^{-\frac{\Gamma_{i}}{2}\left(t-t^{\prime}\right)} E\left(t^{\prime}\right) \alpha_{0}\left(t^{\prime}\right)\right\} .
\end{aligned}
$$

Using the slowly varying envelope expansion of the electric field [Eq. (2)] and applying the rotating wave approximation, we get

$$
\begin{aligned}
\dot{p}_{0}(t)= & -2 \operatorname{Re}\left\{\alpha_{0}^{*}(t) E(t) \sum_{i, a}\left|z_{a, i}\right|^{2} e^{-i \omega_{0} t}\right. \\
& \left.\times \int_{-\infty}^{t} d t^{\prime} e^{i\left(\epsilon_{i}-\epsilon_{a}+\omega_{0}\right)\left(t-t^{\prime}\right)} e^{-\frac{\Gamma_{i}}{2}\left(t-t^{\prime}\right)} \varepsilon_{0}\left(t^{\prime}\right) e^{i \phi\left(t^{\prime}\right)} \alpha_{0}\left(t^{\prime}\right)\right\} .
\end{aligned}
$$

The bandwidth of the FEL will give rise to a photoelectron spectral linewidth of the order of several $\mathrm{eV}$. This range is narrow enough so that the matrix elements $z_{a, i}$ are practically constant at high $\omega_{0}$. Thus, when integrating over the continuum $\left(\sum_{a}\right)$, the factor $e^{-i \epsilon_{a}\left(t-t^{\prime}\right)}$ in the integrand of Eq. (A5) gives rise to an effective delta function with respect to $\left(t-t^{\prime}\right)$. The terms $\varepsilon_{0}\left(t^{\prime}\right) e^{i \phi\left(t^{\prime}\right)} \alpha_{0}\left(t^{\prime}\right)$ can hence be evaluated at $t=t^{\prime}$ and drawn out of the integral. Equation (A5) then becomes

$$
\begin{aligned}
\dot{p}_{0}(t)= & -2 \operatorname{Re}\left\{\left|\alpha_{0}(t)\right|^{2} E(t) \varepsilon_{0}(t) e^{i \phi(t)} e^{-i \omega_{0} t}\right. \\
& \left.\times \sum_{i, a}\left|z_{a, i}\right|^{2} \frac{1}{i\left(\epsilon_{a}-\epsilon_{i}-\omega_{0}-i \frac{\Gamma_{i}}{2}\right)}\right\} .
\end{aligned}
$$

After averaging over an x-ray optical cycle we find

$$
\left\langle E(t) \varepsilon_{0}(t) e^{i \phi(t)} e^{-i \omega_{0} t}\right\rangle=\frac{1}{2} \varepsilon_{0}(t)^{2} .
$$

With the expression of the cycle-averaged flux $J(t)$ given by Eq. (17) we therefore get

$$
\begin{aligned}
\dot{p}_{0}(t)= & -p_{0}(t) J(t) 8 \pi^{2} \alpha \omega_{0} \\
& \times \sum_{i, a}\left|z_{a, i}\right|^{2} \frac{1}{\pi} \frac{\frac{\Gamma_{i}}{2}}{\left(\epsilon_{a}-\epsilon_{i}-\omega_{0}\right)^{2}+\frac{\Gamma_{i}^{2}}{4}} .
\end{aligned}
$$

The expression in the second line of Eq. (A8) is equal to the total photoabsorption cross section $\sigma_{\text {tot }}$ and we recover Eq. (18).

Along similar lines we derive the equations of motion for the reduced two-time density matrix [Eq. (15)]. The partial derivative with respect to the first time argument of the reduced 
density matrix defined in Eq. (14) is given by

$$
\frac{\partial}{\partial t} \rho_{i, j}\left(t, t^{\prime}\right)=\sum_{a} \dot{\alpha}_{i}^{a}(t) \alpha_{j}^{a *}\left(t^{\prime}\right)
$$

Inserting the equations determining the evolution of the wavefunction expansion coefficient $\alpha_{i}^{a}$ [Eq. (8)] into Eq. (A9) leads to

$$
\begin{aligned}
\frac{\partial}{\partial t} \rho_{i, j}\left(t, t^{\prime}\right)= & -\frac{\Gamma_{i}}{2} \rho_{i, j}\left(t, t^{\prime}\right)+i \sum_{k} e^{i\left(\epsilon_{k}-\epsilon_{i}\right) t} \rho_{k, j}\left(t, t^{\prime}\right) z_{k, i} E(t) \\
& -i \sqrt{2} E(t) \alpha_{0}(t) \sum_{a} e^{i\left(\epsilon_{a}-\epsilon_{i}\right) t} z_{a, i} \alpha_{j}^{a *}\left(t^{\prime}\right) .
\end{aligned}
$$

The first term in Eq. (A10) describes the Auger decay of the core-excited states (in case that $i=1 s$; otherwise, $\Gamma_{i}=0$ ), the second term the hole-coupling through the resonant field, and the last term reflects the population of the ionic states by ionization of the ground state. The expansion coefficient $\alpha_{j}^{a *}\left(t^{\prime}\right)$ in the last term of Eq. (A10) can be approximated in first order of the electric-field interaction by formal integration of the complex conjugate of Eq. (8):

$\alpha_{j}^{a *}\left(t^{\prime}\right) \approx i \int_{-\infty}^{t^{\prime}} d t^{\prime \prime} e^{-\frac{\Gamma_{j}}{2}\left(t^{\prime}-t^{\prime \prime}\right)} \alpha_{0}^{*}\left(t^{\prime \prime}\right) e^{-i\left(\epsilon_{a}-\epsilon_{j}\right) t^{\prime \prime}} E\left(t^{\prime \prime}\right) \sqrt{2} z_{a, j}^{*}$

Applying the rotating wave approximation in Eq. (A10) and noticing similar to the case of the derivation of the ground-state evolution that the integral over continuum states and the fast oscillating exponential $e^{i \epsilon_{a}\left(t-t^{\prime \prime}\right)}$ will act as a delta function with respect to $t-t^{\prime \prime}$, we get

$$
\begin{aligned}
\frac{\partial}{\partial t} \rho_{i, j}\left(t, t^{\prime}\right)= & -\frac{\Gamma_{i}}{2} \rho_{i, j}\left(t, t^{\prime}\right)+i \sum_{k} e^{i\left(\epsilon_{k}-\epsilon_{i}\right) t} \rho_{k, j}\left(t, t^{\prime}\right) z_{k, i} E(t) \\
& +\left|\alpha_{0}(t)\right|^{2} E(t) \varepsilon_{0}(t) e^{-i \phi(t)} \sum_{a} z_{a, i} z_{a, j}^{*} \\
& \times \frac{e^{i\left(\epsilon_{a}-\epsilon_{i}\right) t} e^{-i\left(\epsilon_{a}-\epsilon_{j}-\omega_{0}\right) t^{\prime}}}{-i\left(\epsilon_{a}+i \frac{\Gamma_{j}}{2}-\epsilon_{j}-\omega_{0}\right)} .
\end{aligned}
$$

We now consider the case $i=j=2 p_{z}$ :

$$
\begin{aligned}
\frac{\partial}{\partial t} \rho_{2 p_{z}, 2 p_{z}}\left(t, t^{\prime}\right)= & i e^{i\left(\epsilon_{1 s}-\epsilon_{2 p_{z}}\right) t} \rho_{1 s, 2 p_{z}}\left(t, t^{\prime}\right) z_{1 s, 2 p_{z}} E(t) \\
& +i\left|\alpha_{0}(t)\right|^{2} E(t) \varepsilon_{0}(t) e^{-i \phi(t)} e^{i \omega_{0} t} \\
& \times \lim _{\Gamma \rightarrow 0+} \sum_{a}\left|z_{a, 2 p_{z}}\right|^{2} \frac{e^{i\left(\epsilon_{a}-\epsilon_{2 p_{z}}-\omega_{0}\right)\left(t-t^{\prime}\right)}}{\left(\epsilon_{a}-\epsilon_{2 p_{z}}-\omega_{0}+i \frac{\Gamma}{2}\right)} .
\end{aligned}
$$

In the following we consider the first term in Eq. (A13). Applying the rotating wave approximation, introducing the detuning from the resonance

$$
\delta:=\epsilon_{2 p_{z}}-\epsilon_{1 s}-\omega_{0},
$$

and the complex Rabi frequency

$$
R(t)=z_{1 s, 2 p_{z}} \varepsilon_{0}(t) e^{i \phi(t)},
$$

the first term in Eq. (A13) can be rewritten as

$$
i e^{-i \delta t} \frac{R^{*}(t)}{2} \rho_{1 s, 2 p_{z}}\left(t, t^{\prime}\right) \text {. }
$$

The second term in Eq. (A13) can be reduced by using

$$
\lim _{\epsilon \rightarrow 0^{+}} \frac{1}{x+i \epsilon}=\mathcal{P} \mathcal{V} \frac{1}{x}-i \pi \delta(x) \text {. }
$$

Assuming that $\left|z_{a, 2 p) z}\right|^{2}$ varies slowly over the photoelectron energy range of interest, the principal value integral vanishes and the second term of Eq. (A13) becomes

$$
\begin{aligned}
& i\left|\alpha_{0}(t)\right|^{2} E(t) \varepsilon_{0}(t) e^{-i \phi(t)} e^{i \omega_{0} t} \\
& \quad \times \sum_{a}\left|z_{a, 2 p_{z}}\right|^{2}(-i \pi) \delta\left(\epsilon_{a}-\epsilon_{2 p_{z}}-\omega_{0}\right) .
\end{aligned}
$$

Averaging over an optical cycle and realizing that the photoionization cross section of orbital $2 p_{z}$ is given by

$$
\sigma_{2 p_{z}}=\frac{8 \pi^{2}}{c} \omega_{0} \sum_{a}\left|z_{a, 2 p_{z}}\right|^{2} \delta\left(\epsilon_{a}-\epsilon_{2 p_{z}}-\omega_{0}\right)
$$

Eq. (A13) finally reduces to

$$
\frac{\partial}{\partial t} \rho_{2 p_{z}, 2 p_{z}}=\frac{\sigma_{2 p_{z}}}{2} J(t) p_{0}(t)+i e^{-i \delta t} \frac{R^{*}(t)}{2} \rho_{1 s, 2 p_{z}} .
$$

The other equations for the reduced density matrix Eqs. (15) are derived in a similar way.
[1] W. Ackermann et al., Nat. Photon. 1, 336 (2007).

[2] P. Emma et al., Nat. Photon. 4, 641 (2010).

[3] T. Ishikawa et al., Nat. Photon. 6, 540 (2012).

[4] L. Young et al., Nature (London) 466, 56 (2010).

[5] G. Doumy et al., Phys. Rev. Lett. 106, 083002 (2011).

[6] E. Kanter et al., Phys. Rev. Lett. 107, 233001 (2011).

[7] N. Rohringer et al., Nature (London) 481, 488 (2012).

[8] N. Rohringer and R. Santra, Phys. Rev. A 77, 053404 (2008).

[9] J.-C. Liu, Y.-P. Sun, C.-K. Wang, H. Ågren, and F. Gel'mukhanov, Phys. Rev. A 81, 043412 (2010).

[10] P. V. Demekhin and L. S. Cederbaum, Phys. Rev. A 83, 023422 (2011).
[11] P. V. Demekhin, Y.-C. Chiang, and L. S. Cederbaum, Phys. Rev. A 84, 033417 (2011).

[12] L. A. A. Nikolopoulos, T. J. Kelly, and J. T. Costello, Phys. Rev. A 84, 063419 (2011).

[13] K. Rzaznewski, J. Zakrzewski, M. Lewenstein, and J. W. Haus, Phys. Rev. A 31, 2995 (1985).

[14] B. Mollow, Phys. Rev. A 5, 2217 (1972).

[15] V. Schmidt, Electron Spectrometry of Atoms Using Synchrotron Radiation (Cambridge University Press, Cambridge, UK, 1997).

[16] R. Santra and L. S. Cederbaum, Phys. Rep. 368, 1 (2002).

[17] P. V. Demekhin, S. D. Stoychev, A. I. Kuleff, and L. S. Cederbaum, Phys. Rev. Lett. 107, 273002 (2011). 
[18] S.-K. Son and R. Santra, XATOM - An Integrated Toolkit for $X$-ray and Atomic Physics (CFEL, DESY, Hamburg, Germany, 2011).

[19] S.-K. Son, L. Young, and R. Santra, Phys. Rev. A 83, 033402 (2011).

[20] E. Goulielmakis et al., Nature (London) 466, 739 (2010).

[21] N. Rohringer and R. Santra, Phys. Rev. A 79, 053402 (2009).

[22] Y. Li, S. Krinsky, J. W. Lewellen, K.-J. Kim, V. Sajaev, and S. V. Milton, Phys. Rev. Lett. 91, 243602 (2003).

[23] S. Krinsky and R. L. Gluckstern, Phys. Rev. ST Accel. Beams 6, 050701 (2003).

[24] C. Gutt, P. Wochner, B. Fischer, H. Conrad, M. Castro-Colin, S. Lee, F. Lehmkühler, I. Steinke, M. Sprung, W. Roseker, D. Zhu, H. Lemke, S. Bogle, P. H. Fuoss, G. B. Stephenson,
M. Cammarata, D. M. Fritz, A. Robert, and G. Grübel, Phys. Rev. Lett. 108, 024801 (2012).

[25] N. Rohringer and R. Santra, Phys. Rev. A 76, 033416 (2007).

[26] M. C. Teich and G. J. Wolga, Phys. Rev. Lett. 16, 625 (1966).

[27] P. Avan and C. Cohen-Tannoudji, J. Phys. B 10, 155 (1977).

[28] J. H. Eberly, Phys. Rev. Lett. 37, 1387 (1976).

[29] P. Zoller, J. Phys. B 11, 2825 (1978).

[30] H. J. Kimble and L. Mandel, Phys. Rev. A 15, 689 (1977).

[31] G. S. Agarwal, Phys. Rev. Lett. 37, 1383 (1976).

[32] R. Saxena and G. S. Agarwal, J. Phys. B 12, 1939 (1979).

[33] J. C. Camparo and P. Lambropoulos, Phys. Rev. A 47, 480 (1993).

[34] Y. S. Oseledchik and A. I. Bursthein, Radiophys. Quantum Electron. 26, 514 (1983). 